\title{
Elevated serum aspartate aminotransferase levels concomitant with normal alanine aminotransferase levels in older low body weight people: Preliminary findings from a community-based epidemiological study
}

Michi Shibata, Kei Nakajima

\section{Background}

Serum enzyme levels, including hepatic transaminase, are unknown in older people with low body weight (LBW), who can easily experience sarcopenia. Therefore, we addressed preliminarily this issue in a cross-sectional study of an apparently healthy population.

Methods. We investigated the relationship of serum aspartate aminotransferase (AST), alanine aminotransferase (ALT), gamma glutamyl transpeptidase (GGT), alkaline phosphatase (ALP), lactate dehydrogenase (LDH), and total bilirubin levels with body mass index (BMI) and age in 79,623 subjects aged 20-80 years who underwent an annual checkup. The relationship between serum AST and serum creatinine, a surrogate marker of skeletal muscle mass, was also examined in 25,220 subjects who had data for serum creatinine.

Results. Serum levels of AST, ALP, and LDH levels were significantly higher in older $(\geq 50$ years) non-obese subjects compared with younger (< 50 years) corresponding subjects. Serum AST levels were significantly higher in older LBW subjects $\left(B M I \leq 18.9 \mathrm{~kg} / \mathrm{m}^{2}\right.$ ) than in those with a reference BMI of 20.9-22.9 $\mathrm{kg} / \mathrm{m}^{2}$. Serum AST levels showed a J-shaped curve against BMI, whereas ALT and GGT levels showed a linear relationship, regardless of age. Serum levels of creatinine were significantly decreased across the increasing serum AST in men regardless of estimated glomerular filtration rate (eGFR) and women with eGFR $\geq 60$ $\mathrm{mL} / \mathrm{min} / 1.73 \mathrm{~m}^{2}(\mathrm{p}<0.0001)$.

\section{Conclusion}

Elevated serum AST levels concomitant with normal ALT levels, which might reflect systemic damage of skeletal muscle, may be prevalent in older LBW people. These conditions may have involved skeletal muscle damage. Further studies need to determine 
bioRxiv preprint doi: https://doi.org/10.1101/528034; this version posted August 11,2019 . The copyright holder for this preprint (which was not certified by peer review) is the author/funder, who has granted bioRxiv a license to display the preprint in perpetuity. It is made available under aCC-BY-NC-ND 4.0 International license.

whether such a condition is equivalent to the etiology of sarcopenia. 
1 Elevated serum aspartate aminotransferase levels concomitant with normal alanine

2 aminotransferase levels in older low body weight people: Preliminary findings from a

3 community-based epidemiological study

4

5 Michi Shibata ${ }^{1}$, Kei Nakajima ${ }^{1,2}$

6

$7 \quad{ }^{1}$ School of Nutrition and Dietetics, Faculty of Health and Social Services, Kanagawa University of

8 Human Services, 1-10-1 Heisei-cho, Yokosuka, Kanagawa 238-8522, Japan

$9 \quad{ }^{2}$ Department of Endocrinology and Diabetes, Saitama Medical Center, Saitama Medical University,

101981 Kamoda, Kawagoe, Saitama 350-8550, Japan

11

12

13 Corresponding author: Kei Nakajima, M.D., Ph.D.

14 School of Nutrition and Dietetics, Faculty of Health and Social Services, Kanagawa University of

15 Human Services, 1-10-1 Heisei-cho, Yokosuka, Kanagawa 238-8522, Japan.

16

E-mail: nakajima-rsh@ @uhs.ac.jp; tel: +81-046-828-2660

17

18 


\section{Abstract}

2 Background. Serum enzyme levels, including hepatic transaminase, are unknown in older

3 people with low body weight (LBW), who can easily experience sarcopenia. Therefore, we

4 addressed preliminarily this issue in a cross-sectional study of an apparently healthy

5 population.

6 Methods. We investigated the relationship of serum aspartate aminotransferase (AST),

7 alanine aminotransferase (ALT), gamma glutamyl transpeptidase (GGT), alkaline

8 phosphatase (ALP), lactate dehydrogenase (LDH), and total bilirubin levels with body

9 mass index (BMI) and age in 79,623 subjects aged 20-80 years who underwent an annual

10 checkup. The relationship between serum AST and serum creatinine, a surrogate marker of

11 skeletal muscle mass, was also examined in 25,220 subjects who had data for serum

12 creatinine.

13 Results. Serum levels of AST, ALP, and LDH levels were significantly higher in older ( $\geq 50$

14 years $)$ non-obese subjects compared with younger $(<50$ years $)$ corresponding subjects.

15 Serum AST levels were significantly higher in older LBW subjects $\left(\mathrm{BMI} \leq 18.9 \mathrm{~kg} / \mathrm{m}^{2}\right)$ than

16 in those with a reference BMI of 20.9-22.9 kg/m². Serum AST levels showed a J-shaped

17 curve against BMI, whereas ALT and GGT levels showed a linear relationship, regardless

18 of age. Serum levels of creatinine were significantly decreased across the increasing serum

19 AST in men regardless of estimated glomerular filtration rate (eGFR) and women with

$20 \quad \mathrm{eGFR} \geq 60 \mathrm{~mL} / \mathrm{min} / 1.73 \mathrm{~m}^{2}(\mathrm{p}<0.0001)$.

21 Conclusion. Elevated serum AST levels concomitant with normal ALT levels, which might

22 reflect systemic damage of skeletal muscle, may be prevalent in older LBW people. These

23 conditions may have involved skeletal muscle damage. Further studies need to determine

24 whether such a condition is equivalent to the etiology of sarcopenia. 
1 Key words: AST, ALT, BMI, low body weight, older people

2

\section{Introduction}

4 Whether serum transaminase levels are decreased or increased in individuals with

5 low-body weight (LBW) is unclear. In the geriatric population, LBW individuals are at

6 increased risk for sarcopenia, an age-associated loss of muscle mass and function, which

7 lead to disability, hospitalization, and death (Fielding et al. 2011, Calvani et al. 2015).

8 Aspartate aminotransferase (AST), alanine aminotransferase (ALT), and gamma glutamyl

9 transpeptidase (GGT), which are routinely measured in an ordinary checkup, are present in

10 multiple organs, including the liver, skeletal muscle, heart, and kidney (Lott and

11 Landesman. 1984, Nathwani at al. 2005, Malakouti et al. 2017). However, ALT and GGT

12 are predominantly found in the liver. Therefore, elevated serum AST levels concomitant

13 with normal serum ALT levels could reflect injury of skeletal muscle and myocardium in

14 clinical practice (Nathwani at al. 2005, Malakouti et al. 2017). Alkaline phosphatase (ALP)

15 and lactate dehydrogenase (LDH) are also found in multiple organs, whereas ALP is

16 preferentially included in bone tissue (Malakouti et al. 2017, Lowe and John. 2018). LDH

17 is involved in similar organs, such the liver and skeletal muscle, where AST is found (Lott

18 and Landesman. 1984, Nathwani at al. 2005, Malakouti et al. 2017).

19 We investigated the relationship of serum levels of AST, ALT, GGT, ALP, LDH, and total

20 bilirubin, a marker of hepatic function (Gazzin et al. 2016), with body mass index (BMI) in

21 an apparently healthy, general population aged from $20-80$ years. To confirm that high

22 serum AST with normal serum ALT can reflect skeletal muscle damage, the relationship

23 between serum AST and serum creatinine, a surrogate marker of skeletal muscle mass

24 (Hosten et al. 1990), was also examined.

\section{Methods}


1 This preliminary cross-sectional study was part of an observational study that was

2 performed to examine the relationships between lifestyle-related diseases and

3 cardiometabolic risk factors (Muneyuki et al. 2013). The current study involved 2

4 institutions in Kanagawa and Saitama, Japan: Kanagawa University of Human Services and

5 the Saitama Health Promotion Corporation, a public interest corporation. The protocol was

6 approved by the Ethics Committees of Kanagawa University of Human Services

7 (No.10-22). Informed consent was obtained from all patients who were included in the

8 study.

\section{Subjects}

11 Clinical data were obtained for 116,817 apparently healthy individuals, who underwent

12 routine check-ups in Saitama Prefecture between April 2007 and March 2008. Inpatients

13 and disabled individuals who could not move without assistance were not enrolled. After

14 also excluding individuals with incomplete data of AST, ALT, and GGT, 79,623 subjects

15 remained in the study (54,190 men and 25,433 women). To investigate the effect of age,

16 subjects were divided into five age groups: $20-29,30-39,40-49,50-59$, and 60-80 years.

17 The relationship between serum AST and serum creatinine was examined in a subgroup of

18 subjects who had data of serum creatinine $(n=25,220)$ after the restriction of subjects with

19 serum ALT of $<30 \mathrm{U} / \mathrm{L}$, which was conducted because of exclusion of high AST due to

20 hepatic damage. 
2 Anthropometry and laboratory assays

3 Anthropometric measurements and the collection of blood samples for laboratory analysis

4 were performed in the morning. BMI was calculated as body weight ( $\mathrm{kg})$ divided by height

5 squared $\left(\mathrm{m}^{2}\right)$. Subjects were divided into six BMI categories $(\leq 18.9,19.0-20.9,21.0-22.9$,

$6 \quad 23.0-24.9,25.0-26.9$, and $\geq 27.0 \mathrm{~kg} / \mathrm{m}^{2}$ ), as previously reported (Muneyuki et al. 2013).

7 Because the prevalence of underweight subjects $\left(\mathrm{BMI}<18.5 \mathrm{~kg} / \mathrm{m}^{2}\right.$ ) in their $50 \mathrm{~s}$ and older

8 was low (3.3\%) in this study, we defined the lowest BMI category as $\leq 18.9 \mathrm{~kg} / \mathrm{m}^{2}$, which

9 was termed as LBW. When we selected these BMI categories, we took into consideration

10 that the World Health Organization proposed that the BMI cutoff points for overweight and

11 obesity in Asian populations should be $\geq 23.0$ and $\geq 27.5 \mathrm{~kg} / \mathrm{m}^{2}$, respectively. These cutoff

12 points are lower than those in Western countries (WHO Expert Consultation. 2004).

13 To evaluate the relationship between serum AST and serum creatinine, serum AST level

14 was classified into three ranges, $<20 \mathrm{U} / \mathrm{L}, 20-29 \mathrm{U} / \mathrm{L}$, and $\geq 30 \mathrm{U} / \mathrm{L}$. The estimated

15 glomerular filtration rate (eGFR) was calculated using the following equation: eGFR

$16\left(\mathrm{ml} / \mathrm{min} / 1.73 \mathrm{~m}^{2}\right)=194 \times$ serum $\mathrm{Cr}-1.094 \times$ age $-0.287($ if female $) \times 0.739$, where $\mathrm{Cr}$ denotes

17 serum creatinine concentration (mg/dL) (Matsuo et al. 2009). The eGFR was divided into

18 two groups: $\geq 60$ and $<60 \mathrm{ml} / \mathrm{min} / 1.73 \mathrm{~m}^{2}$, a criteria for chronic kidney desease because

19 serum creatinine is elevated in patients with renal failure. 
2 Statistical analysis

3 Data in Figure are expressed as means $\square \pm \square$ standard erros. Significant differences in

4 parameteres between BMI categories were evaluated using analysis of variance (ANOVA).

5 Significant differences in serum creatinine between three AST categories were also

6 evaluated using ANOVA and post-hoc Bonferroni test. Differences between subjects with a

7 provisional reference BMI of $21.0-22.9 \mathrm{~kg} / \mathrm{m}^{2}$ (Muneyuki et al. 2013, WHO Expert

8 Consultation. 2004) and other BMI groups, and differences between the five age groups

9 were evaluated using the post-hoc Bonferroni test and the Mann-Whitney test. Statistical

10 analyses were performed using SAS-Enterprise Guide (SAS-EG 7.1; SAS Institute, Cary,

$11 \mathrm{NC}, \mathrm{USA}) . \mathrm{P}<0.05$ was considered to represent statistical significance.

14 All serum enzymes, except for ALP, in subjects aged $\geq 50$ years and LDH in those aged $\geq$

1560 years, significantly increased across the increasing BMI categories (all $\mathrm{P}<0.0001$,

16 ANOVA; Figure 1). AST, GGT, ALP, and LDH levels were significantly higher in older ( $\geq$

1750 years) non-obese subjects (BMI $<25.0 \mathrm{~kg} / \mathrm{m}^{2}$ ) compared with younger $(<50$ years)

18 corresponding subjects (Mann-Whitney test, all P < 0.0001). Notably, serum AST levels in

19 older LBW subjects were significantly higher than those in subjects with a provisional 
1 reference BMI of 20.9-22.9 kg/m² (P=0.0003, Bonferroni test). Consequently, serum AST

2 levels showed a J-shaped curve against BMI in older subjects. However, ALT and GGT

3 levels showed almost a linear relationship against BMI, regardless of age groups. No

4 significant difference was observed in serum total bilirbin levels between BMI and age

5 groups.

6 As shown in Figure 2, serum creatinine was decreased across the increasing AST in men

7 regardless of eGFR and women with eGFR $\geq 60 \mathrm{~mL} / \mathrm{min} / 1.73 \mathrm{~m}^{2}$ (ANOVA, all $\mathrm{p}<0.0001$ ).

8 In Addition, Post-hoc Bonferroni test indicated that serum creatinine in subjects with AST

$9 \geq 30 \mathrm{U} / \mathrm{L}$ was significantly lower than those with AST $\leq 19 \mathrm{U} / \mathrm{L}$ (all p <0.0001).

11 Discussion

12 To date, prevention of sarcopenia has been the focus of attention in aging societies

13 (Fielding et al. 2011, Calvani et al. 2015). However, there is no blood marker for

14 sarcopenia. A blood marker might enable us to detect sarcopenia earlier in the general

15 population because the current definition of sarcopenia may be complicated and

16 time-consuming to determine (Fielding et al. 2011, Calvani et al. 2015). The current

17 preliminary study showed that LBW subjects in their $\geq 50$ s had elevated serum AST levels,

18 which were acompanied by high ALP and LDH levels, but low to normal serum ALT levels, 
1 compared with coresponding younger subjects and those with a reference BMI. To the best

2 of our knowledge, this is the first report to show such an observation. Although all enzymes

3 measured in this study are likely present in multiple organs, ALT and GGT are

4 predominantly found in the liver (Lott and Landesman. 1984, Nathwani at al. 2005,

5 Malakouti et al. 2017). Therefore, elevated AST concomitant with normal ALT levels,

6 resulting in a dissociation between serum AST and ALT levels in older LBW subjects,

7 suggests non-hepatic injury. This could reflect systemic damage of skeletal muscle mass,

8 including the myocadium. Some studies have reported that low ALT levels may be

9 associated with sarcopenia (Ruhl and Everhart. 2013, Vespasiani- Gentilucci et al. 2018)

10 This is partially consistent with our results because LBW subjects in our study had low to

11 normal ALT levels compared with normal weight and obese subjects (Figure 1). High

12 serum ALP levels in older LBW subjects may be attributable to concurrently occurring

13 damage in bone tissue (Malakouti et al. 2017, Lowe and John. 2018). This possibility

14 deserves further study. Unfortunately, isoforms of enzymes and serum creatinine kinase

15 were unavailable in this study, which is a major limitation of this study. Additionally, other

16 conditions that only elicit elevated AST levels were not thoroughly excluded.

17 Although circulating creatine kinase, aldolase, and myoglobin are indicators of skeletal

18 muscle damage, these parameters are only measured in the clinical setting if a patient

19 complains of muscle pain or the physician suspects myositis. Therefore, in the current study, 
1 we confirmed the inverse relationship between serum creatinine and serum AST in the

2 subjects without high ALT, suggesting a rough trend that skeletal muscle mass is reduced in

3 individuals with high AST and normal ALT.

4 In conclusion, older LBW people may have elevated AST comcomitant with normal ALT

5 levels, compared with corresponding younger people and those with the reference BMI.

6 These conditions may have involved skeletal muscle damage. Further study is required to

7 determine whether this specific condition is equivalent to the etiology of sarcopenia.

8

9 Acknowledgments

10 None

11

12 Declaration of Conflicting Interests

13 The authors declare that there is no conflict of interest.

14

15 Supportive foundations

16 None

17

18 Author contributions

19 Shibata $\mathrm{M}$ and Nakajima $\mathrm{K}$ designed the study and analyzed the data. Nakajima K wrote

20 the manuscript. Both authors have read and approved the final manuscript. 


\section{References}

Calvani R, Marini F, Cesari M, Tosato M, Anker SD, von Haehling S, Miller RR, Bernabei R, Landi F, Marzetti E; SPRINTT consortium. 2015. Biomarkers for physical frailty and sarcopenia: state of the science and future developments. J Cachexia Sarcopenia Muscle. 6:278-86. doi: 10.1002/jcsm.12051.

Fielding RA, Vellas B, Evans WJ, et al. 2011. Sarcopenia: an undiagnosed condition in older adults. Current consensus definition: prevalence, etiology, and consequences. International working group on sarcopenia. J Am Med Dir Assoc. 12:249-56. doi: 10.1016/j.jamda.2011.01.003.

Gazzin S, Vitek L, Watchko J, Shapiro SM, Tiribelli C. 2016. A Novel Perspective on the Biology of Bilirubin in Health and Disease. Trends Mol Med. 22:758-768. doi: 10.1016/j.molmed.2016.07.004.

Hosten AO. BUN and Creatinine. In: Walker HK, Hall WD, Hurst JW, editors. Clinical Methods: The History, Physical, and Laboratory Examinations. 3rd edition. Boston: Butterworths; 1990. Chapter 193.

Lott JA, Landesman PW. The enzymology of skeletal muscle disorders. 1984. Crit Rev Clin Lab Sci. 20:153-90. DOI: 10.3109/10408368409165773.

Lowe D, John S. Alkaline Phosphatase. 2018. StatPearls [Internet]. Treasure Island (FL): StatPearls Publishing; 2018-.

Malakouti M, Kataria A, Ali SK, Schenker S. 2017. Elevated Liver Enzymes in Asymptomatic Patients - What Should I Do? J Clin Transl Hepatol. 5:394-403. doi: 10.14218/JCTH.2017.00027.

Muneyuki T, Suwa K, Oshida H, et al. 2013. Design of the Saitama Cardiometabolic Disease and Organ Impairment Study (SCDOIS): A multidisciplinary observational epidemiological study. Open J Endocr Metab Dis. 2:144-56. DOI: 
2 Matsuo S, Imai E, Horio $\mathrm{M}$, et al. Revised equations for estimated GFR from serum $3 \quad$ creatinine in Japan. Am J Kidney Dis 2009;53:982-92.

4 Nathwani RA, Pais S, Reynolds TB, Kaplowitz N. 2005. Serum alanine aminotransferase

$5 \quad$ in skeletal muscle diseases. Hepatology. 41:380-2. DOI: 10.1002/hep.20548

6 Ruhl CE, Everhart JE. 2013. The association of low serum alanine aminotransferase activity with mortality in the US population. Am J Epidemiol. 178:1702-11. doi: 10.1093/aje/kwt209.

9 Vespasiani-Gentilucci U, De Vincentis A, Ferrucci L, Bandinelli S, Antonelli Incalzi R,

WHO Expert Consultation. 2004. Appropriate body-mass index for Asian populations and

\section{Figure legend}

\section{Figure 1. Serum enzyme and total birlibin levels according to BMI and age groups}

19 The symbols indicate the mean values of parameters. The vertical bars represent the

20 standard error. BMI categories of one to six represent $\leq 18.9,19.0-20.9,21.0-22.9$,

$2123.0-24.9,25.0-26.9$, and $\geq 27.0 \mathrm{~kg} / \mathrm{m}^{2}$. The number of subjects was $1848,1745,1268,971$,

22 and 384 in 20s, 30s, 40s, 50s, and 60-80 years old, 3694, 3773, 3160, 2768, and 873 in 20s,

23 30s, 40s, 50s, and 60-80 years old, 4039, 4741, 4308, 4436, and 1541 in 20s, 30s, 40s, 50s, 
1 and 60-80 years old, 2683, 3712, 4229, 4627, and 1656 in 20s, 30s, 40s, 50s, and 60-80

2 years old, 1465, 2454, 2990, 3401, and 1141 in 20s, 30s, 40s, 50s, and 60-80 years old,

$31564,3065,3465,2935$, and 687 in 20 s, 30s, 40s, 50s, and $60-80$ years old, for the BMI

4 categories from one to six (AST, ALT, and GGT). Data of ALP, LDH, and total bilirubin

5 were available only in $20,773,7050$, and 9061 subjects in total, respectively.

6

7 Figure 2. Serum creatinine levels according to three serum AST categories

8 The symbols indicate the mean values of parameters. The vertical bars represent the

9 standard error. Open and closed circles represent subjects with eGFR $\geq 60 \mathrm{~mL} / \mathrm{min} / 1.73 \mathrm{~m}^{2}$

10 and subjects with eGFR $<60 \mathrm{~mL} / \mathrm{min} / 1.73 \mathrm{~m}^{2}$, respectively. $* \mathrm{P}<0.0001$, Bonferroni test.

11 The number of subjects was 3268,4430 , and 343 in men with eGFR $\geq 60 \mathrm{~mL} / \mathrm{min} / 1.73 \mathrm{~m}^{2}$,

12 and 2500, 4629, and 411 in men with eGFR $<60 \mathrm{~mL} / \mathrm{min} / 1.73 \mathrm{~m}^{2}$, and 60-80 years old,

13 and 2210, 1212, and 46 in women with eGFR $\geq 60 \mathrm{~mL} / \mathrm{min} / 1.73 \mathrm{~m}^{2}$, and 2987, 2967, and

14 217, for the three AST categories.

15 

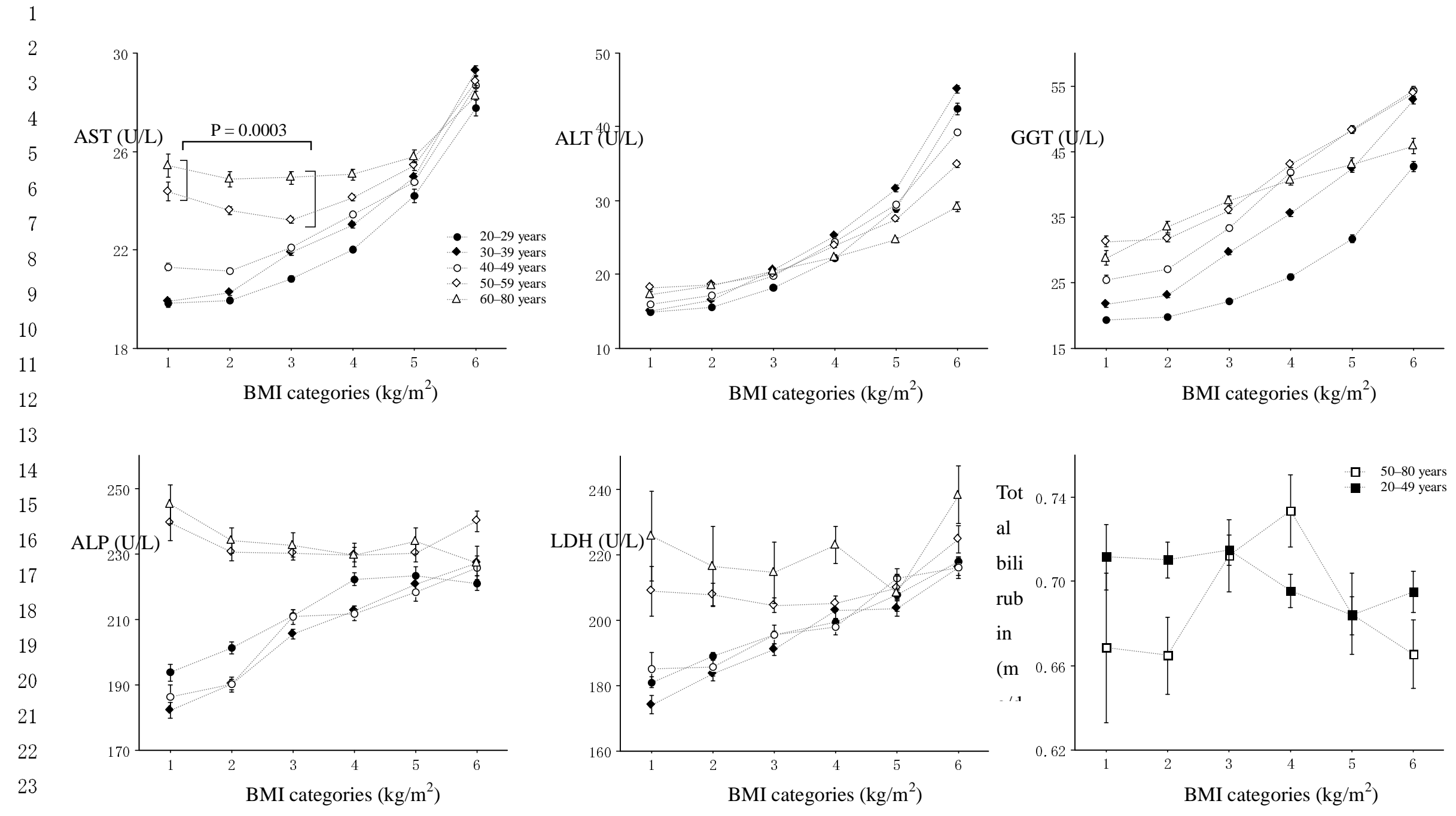

Figure 1 Enzymes and BMI categories 
Men

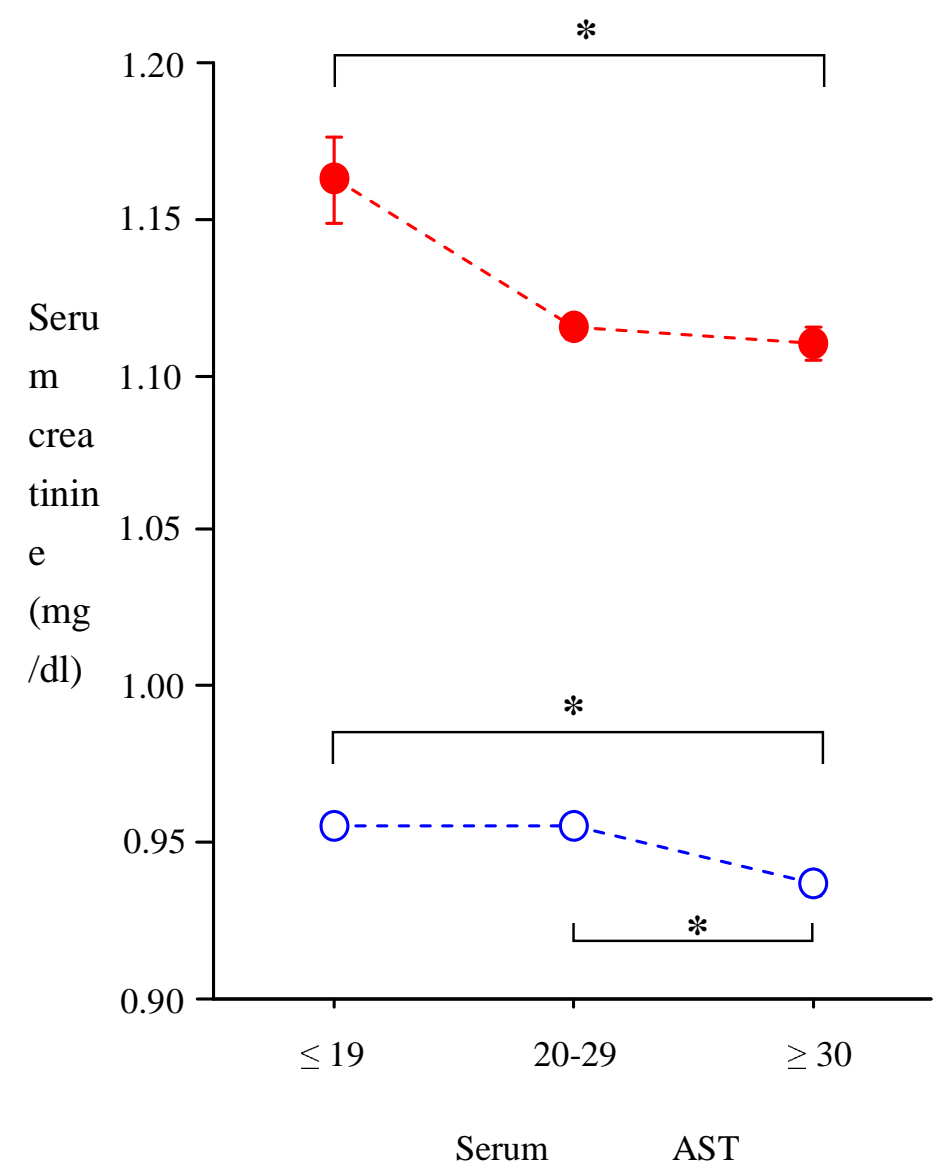

Women

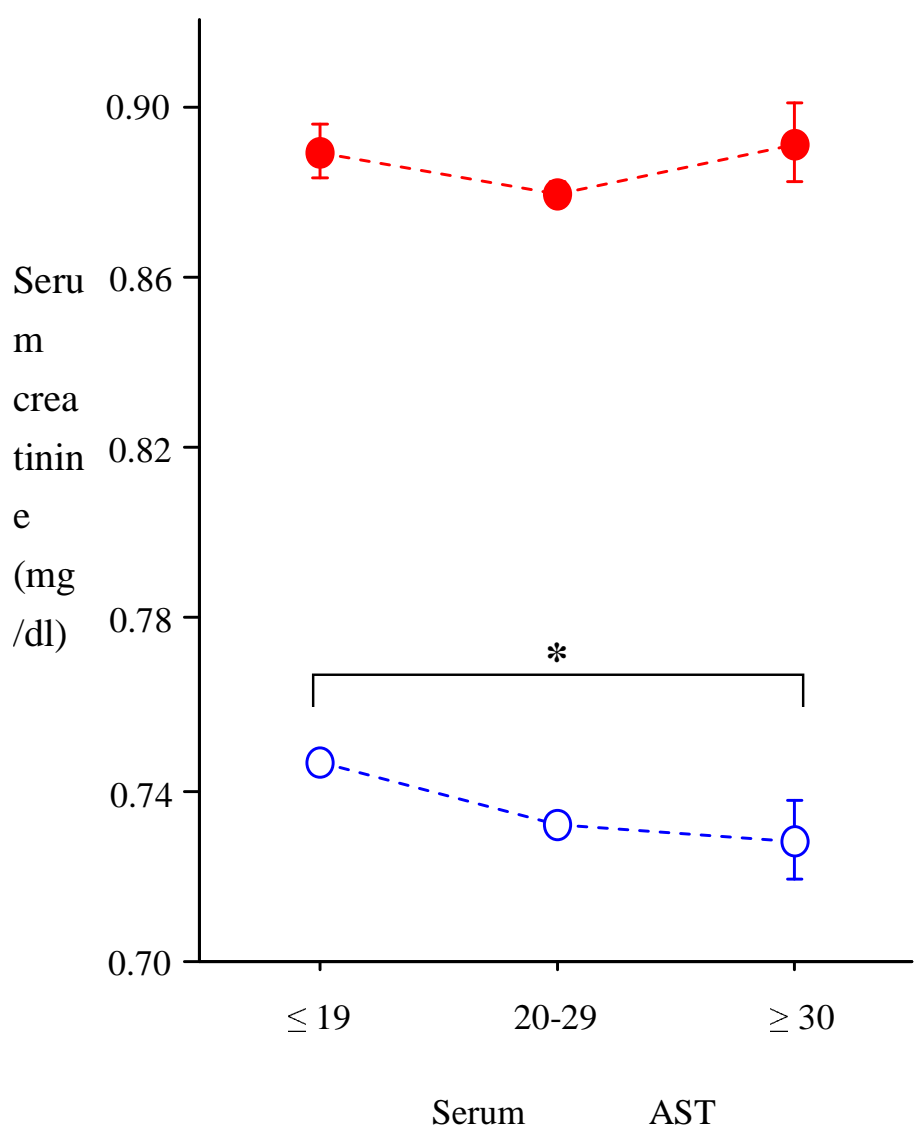

Figure 2 Serum Creatinine and serum AST categories 